\title{
ON ISOMETRIC EMBEDDINGS OF GRAPHS
}

BY

\author{
R. L. GRAHAM AND P. M. WINKLER
}

\begin{abstract}
If $G$ is a finite connected graph with vertex set $V$ and edge set $E$, a standard way of defining a distance $d_{G}$ on $G$ is to define $d_{G}(x, y)$ to be the number of edges in a shortest path joining $x$ and $y$ in $V$. If $\left(M, d_{M}\right)$ is an arbitrary metric space, then an embedding $\lambda: V \rightarrow M$ is said to be isometric if $d_{G}(x, y)=$ $d_{M}(\lambda(x), \lambda(y))$ for all $x, y \in V$. In this paper we will lay the foundation for a theory of isometric embeddings of graphs into cartesian products of metric spaces.
\end{abstract}

Introduction. With a finite connected undirected $\operatorname{graph}^{1} G=(V, E)$ one can associate a metric $d_{G}: V \times V \rightarrow \mathbf{N}$ (the set of nonnegative integers) by defining $d_{G}(x, y)$ to be the number of edges in a shortest path between $x$ and $y$ for all $x, y \in V$, the vertex set of $G$. If $\left(M, d_{M}\right)$ is an arbitrary metric space we say that an embedding $\lambda: V \rightarrow M$ is isometric $^{2}$ if, for all $x, y \in V$,

We denote this by writing $\lambda: V \stackrel{I}{\rightarrow} M$.

$$
d_{M}(\lambda(x), \lambda(y))=d_{G}(x, y) \text {. }
$$

A number of papers have appeared in the past few years which deal with various properties of graphs that have isometric embeddings in certain metric and semimetric $^{3}$ spaces (e.g., see [As1, As2, As3, ADe1, ADe2, ADz1, ADz2, Av1, Av2, BGK, Dew, Dez1, Dez2, Dj, F, GP1, GP2, K1, K2, K3, Y]).

In this paper we will lay the foundation for a theory of isometric embedding of graphs into cartesian products of metric spaces. Although our primary focus will be on embeddings into products of graphs we should remark that many of the results actually extend to products of more general metric and semimetric spaces.

Embedding into cartesian products. For a connected graph $G=(V, E)$ define a relation $^{4} \theta$ on $E$ as follows: If $e=\{x, y\} \in E$ and $e^{\prime}=\left\{x^{\prime}, y^{\prime}\right\} \in E$, then $e \theta e^{\prime}$ if

$$
d_{G}\left(x, x^{\prime}\right)+d_{G}\left(y, y^{\prime}\right) \neq d_{G}\left(x, y^{\prime}\right)+d_{G}\left(x^{\prime}, y\right) \text {. }
$$

The relation $\theta$ is easily seen to be well defined, reflexive and symmetric; let $\hat{\theta}$ be its transitive closure, and let $E_{i}, 1 \leqslant i \leqslant r$, be the equivalence classes of $\hat{\theta}$. Thus, $E=\bigcup_{i=1}^{r} E_{i}$.

For each $i, 1 \leqslant i \leqslant r$, let $G_{i}$ denote the graph $\left(V, E \backslash E_{i}\right)$ and let $C_{i}(1), C_{i}(2), \ldots, C_{i}\left(m_{i}\right)$ denote the connected components of $G_{i}$. Finally, form the

Received by the editors January $5,1984$.

1980 Mathematics Subject Classification.Primary 05C50, 54E40.

${ }^{1}$ In general, we follow the terminology of [BM].

${ }^{2}$ In the literature this is also sometimes said to be "distance preserving".

${ }^{3}$ I.e., the triangle inequality may fail (in French, écart).

${ }^{4}$ First introduced in [Dj]. 
graphs $G_{i}^{*}=\left(V_{i}^{*}, E_{i}^{*}\right), 1 \leqslant i \leqslant r$, by letting $V_{i}^{*}=\left\{C_{i}(1), \ldots, C_{i}\left(m_{i}\right)\right\}$ and taking $\left\{C_{i}(j), C_{i}\left(j^{\prime}\right)\right\}$ to be an edge of $G_{i}^{*}$ iff some edge in $E_{i}$ joins a point in $C_{i}(j)$ to a point in $C_{i}\left(j^{\prime}\right)$. For $v \in C_{i}(j)$, denote by $\alpha_{i}: V \rightarrow V_{i}^{*}$ the natural contraction $v \rightarrow C_{i}(j) \in V_{i}^{*}$.

Next, define an embedding $\alpha: G \rightarrow \prod_{i=1}^{r} G_{i}^{*}$, which we will call the canonical embedding of $G$, by $\alpha(v)=\left(\alpha_{1}(v), \alpha_{2}(v), \ldots, \alpha_{r}(v)\right)$.

THEOREM 1. The canonical embedding $\alpha: G \rightarrow \prod_{i=1}^{r} G_{i}^{*}$ is isometric.

Proof. Let $x_{0}$ and $x_{k}$ be a pair of vertices of $G$ with $d_{G}\left(x_{0}, x_{k}\right)=k$. For brevity we will henceforth usually denote $d_{G}(x, y)$ by $x y$. Let $\left(\left\{x_{0}, x_{1}\right\}\right.$, $\left.\left\{x_{1}, x_{2},\right\}, \ldots,\left\{x_{k-1}, x_{k}\right\}\right)$ be a minimal path between $x_{0}$ and $x_{k}$, and let $P$ denote the set of edges in this path. For each $i, 1 \leqslant i \leqslant r$, let $p_{i}=\left|P \cap E_{i}\right|$. Thus, $\sum_{i=1}^{r} p_{i}=k$.

FACT. If $Q$ is any other path connecting $x_{0}$ to $x_{k}$, then $\left|Q \cap E_{i}\right| \geqslant p_{i}$ for all $i$.

ProOF OF FACT. For each $j, 1 \leqslant j \leqslant k$, define a function $\alpha_{j}$ on $V$ by $a_{j}(u)=u x_{j}$ - $u x_{j-1}$. Let $b(u)=\sum_{j=1}^{k} a_{j}(u)$ and, for each $i, 1 \leqslant i \leqslant r$, set

$$
b_{i}(u)=\sum\left\{a_{j}(u):\left\{x_{j-1}, x_{j}\right\} \in E_{i}\right\} \text {. }
$$

Then

$$
\sum_{s i=1}^{r} b_{i}(u):=b(u)=u x_{k}-u x_{0} .
$$

If $\{u, v\} \in E$, then $\left|u x_{0}-v x_{0}\right| \leqslant 1$ and $\left|u x_{k}-v x_{k}\right| \leqslant 1$. Hence, $|b(u)-b(v)| \leqslant 2$.

Suppose $\{u, v\} \in E$ and $\left\{x_{j-1}, x_{j}\right\} \notin E_{i}$. Then $u x_{j-1}+v x_{j}=u x_{j}+v x_{j-1}$ by the definition of $\theta$, and so $a_{j}(u)=a_{j}(v)$. It follows that $b_{s}(u)=b_{s}(v)$ for all $s \neq i$. Since $|b(u)-b(v)| \leqslant 2$ we also have $\left|b_{i}(u)-b_{i}(v)\right| \leqslant 2$.

Now, for each $j, 0 \leqslant j \leqslant k$, we have $x_{0} x_{j}=j$ and $x_{j} x_{k}=k-j$. Thus for all $j$ with $1 \leqslant j \leqslant k, a_{j}\left(x_{0}\right)=1$ and $a_{j}\left(x_{k}\right)=-1$. Therefore, for each $i, 1 \leqslant i \leqslant r$, we have $b_{i}\left(x_{0}\right)=p_{i}$ and $b_{i}\left(x_{k}\right)=-p_{i}$.

In following $Q$ from $x_{0}$ to $x_{k}, b_{i}$ must change from $p_{i}$ to $-p_{i}$. However, $b_{i}$ can change by at most 2 along each edge of $Q$ and cannot change at all if the edge does not belong to $E_{i}$. It follows that $\left|Q \cap E_{i}\right| \geqslant\left|p_{i}-\left(-p_{i}\right)\right| \cdot \frac{1}{2}=p_{i}$, and the Fact is proved.

Note in particular, taking $k=1$, that if $\left\{x_{0}, x_{1}\right\} \in E_{i}$, then every path from $x_{0}$ to $x_{1}$ has at least one edge in $E_{i}$ and, consequently, $x_{0}$ and $x_{1}$ belong to different connected components in $G_{i}$, i.e., they are not identified in $G_{i}^{*}$.

It remains only to check that the distance $\alpha\left(x_{0}\right) \alpha\left(x_{k}\right)$ between the images of $x_{0}$ and $x_{k}$ in $\prod_{i=1}^{r} G_{i}^{*}$ is $k$. But in $G_{i}^{*}, 1 \leqslant i \leqslant r$, the distance between the corresponding connected components $C\left(x_{0}\right)$ and $C\left(x_{k}\right)$ is just the minimum, taken over all paths $Q$ in $G$ from $x_{0}$ to $x_{k}$, of $\left|Q \cap E_{i}\right|$. By the Fact this value is $p_{i}$, so that

$$
\alpha\left(x_{0}\right) \alpha\left(x_{k}\right)=\sum_{i=1}^{r} \alpha_{i}\left(x_{0}\right) \alpha_{i}\left(x_{k}\right)=\sum_{i=1}^{r} p_{i}=k
$$

as required. This proves Theorem 1 .

We will define $r$, the number of factors $G_{i}^{*}$ in the canonical embedding of $G$, to be the isometric dimension of $G$, denoted by $\operatorname{dim}_{I}(G)(\mathrm{cf}$. [Dew]). 
An isometric embedding $G \stackrel{I}{\rightarrow} \prod_{i=1}^{m} H_{i}$ will be termed irredundant if each $H_{i}$ has at least two vertices and for all $i, 1 \leqslant i \leqslant m$, and for all $h \in H_{i}, h$ occurs as a coordinate value of the image of some $g \in G$. Note that any $\beta: G \stackrel{I}{\rightarrow} \prod_{i=1}^{m} H_{i}$ can be made irredundant by discarding unused vertices and factors.

Finally, let us call $G$ irreducible if $G \stackrel{I}{\rightarrow} \prod_{i=1}^{m} H_{i}$ always implies $G \stackrel{I}{\rightarrow} H_{i}$ for some $i$.

THEOREM 2. The canonical embedding $\alpha: G \stackrel{I}{\rightarrow} \prod_{i=1}^{r} G_{i}^{*}$ is irredundant, has irreducible factors and has the largest possible number of factors among all irredundant isometric embeddings of $G$.

Proof. Let $\beta: G \stackrel{I}{\rightarrow} \prod_{j=1}^{m} H_{j}, v \rightarrow\left(v_{1}, \ldots, v_{m}\right)$ be an irredundant isometric embed$\operatorname{ding}$ of $G$. If $e=\{x, y\} \in E$, let $\hat{\beta}(e)$ be the unique $j$ for which $x_{j} \neq y_{j}$. Suppose $e^{\prime}=\left\{x^{\prime}, y^{\prime}\right\} \in E$ and $\hat{\beta}\left(e^{\prime}\right) \neq j=\hat{\beta}(e)$. Then

$$
x x^{\prime}-y x^{\prime}=x_{j} x_{j}^{\prime}-y_{j} x_{j}^{\prime}=x_{j} y_{j}^{\prime}-y_{j} y_{j}^{\prime}=x y^{\prime}-y y^{\prime},
$$

so that

$$
x x^{\prime}+y y^{\prime}=x y^{\prime}+y x^{\prime}
$$

i.e., $e$ and $e^{\prime}$ are not related by $\theta$.

It follows that if $e$ and $e^{\prime}$ are arbitrary edges of $E$ with $e \theta e^{\prime}$, then $\hat{\beta}(e)=\hat{\beta}\left(e^{\prime}\right)$. This means that $\hat{\beta}$ is constant on each equivalence class $E_{i}$. This implies that the range of $\hat{\beta}$ is limited to at most cardinality $r=\operatorname{dim}_{I}(G)$, and the third claim of the theorem follows. Note that $m$ can assume any value between 1 and $r$ by combining canonical factors.

The fact that $\alpha$ is irredundant is clear. Suppose a factor $G_{i_{0}}^{*}$ is not irreducible. Then $G_{i_{0}}^{*}$ has an irredundant isometric embedding $\gamma: G_{i_{0}}^{*} \rightarrow \prod_{j=1}^{k} H_{j}$ with $k>1$, from which we obtain an irredundant isometric embedding of $G$ with $\prod_{i=1: i \neq i_{0}}^{r} G_{i}^{*}$ $\times \Pi_{j=1}^{k} H_{j}$ having more than $r$ factors. This contradiction concludes the proof of the theorem.

The preceding remarks immediately imply the following result.

THEOREM 3. The only irredundant isometric embedding of $G$ into a product of $\operatorname{dim}_{I}(G)$ factors is the canonical embedding. Each factor $H_{j}$ of an irredundant isometric embedding $G \stackrel{I}{\rightarrow} \prod_{j=1}^{m} H_{j}$ embeds canonically into a product of $G_{i}{ }^{*}$ 's.

REMARK. It appears from these results that the category of connected graphs and isometric embeddings behaves much like a variety in universal algebra, with subdirect product representation. Relations containing $\hat{\theta}$ play the role of congruences and the maps $\alpha_{i}: G \rightarrow G_{i}^{*}$ become homomorphisms. However, it is not clear at this point that a graph and its metric can be interpreted as an algebra in such a way as to justify the correspondence.

COROLlaRY. $G$ is irreducible if and only if $G$ has a single $\hat{\theta}$-equivalence class.

As an example we show a graph $G$ in Figure 1(a) with the following properties: (i) $G$ is irreducible, i.e., $\operatorname{dim}_{I}(G)=1$. (ii) $G^{\prime}=G-\{e\}$ (shown in Figure 1(b)) has 
(a)

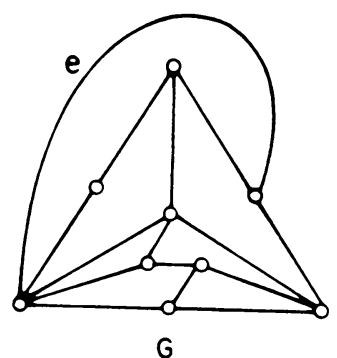

(b)

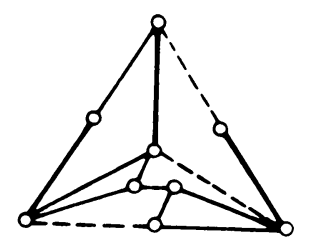

$G^{\prime}=G-\{e\}$

(c)

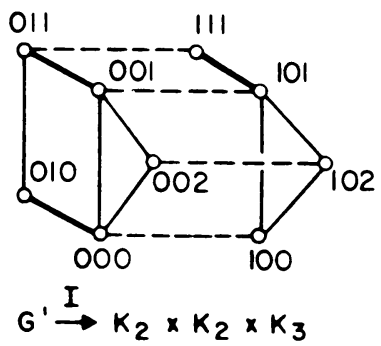

FIGURE 1

$\operatorname{dim}_{I}\left(G^{\prime}\right)=3$. The partition of $E$ into $E_{1}, E_{2}$, and $E_{3}$ is also shown. (iii) In Figure 1(c) we redraw $G^{\prime}$ in a more suggestive form, showing $G^{\prime} \stackrel{I}{\rightarrow} K_{2} \times K_{2} \times K_{3}$.

We remark in closing this section that if we consider the usual random graph model, in which $G=(V, E)$ has $V=\{1,2, \ldots, n\}$ and each pair $\{i, j\}$ is chosen to be an edge with (independent) probability $1 / 2$, then the probability that a random graph with $n$ vertices is irreducible goes to 1 as $n \rightarrow \infty$. In particular, it can be shown by relatively standard probabilistic arguments that almost all graphs on $n$ vertices have the property that, for any two pairs $\{\alpha, \beta\}$ and $\left\{\alpha^{\prime}, \beta^{\prime}\right\}$ of vertices, there is another pair $\{x, y\}$ such that the subgraph induced by these 6 points is that shown in Figure 2. Thus,

$$
\begin{aligned}
\alpha x+\beta y & =2<4=\alpha y+\beta x, \\
\alpha^{\prime} x+\beta y^{\prime} & =2<4=\alpha^{\prime} y+\beta^{\prime} x,
\end{aligned}
$$

so that if $\{\alpha, \beta\}=e \in E$ and $\left\{\alpha^{\prime}, \beta^{\prime}\right\}=e^{\prime} \in E$, then $e \theta\{x, y\}, e^{\prime} \theta\{x, y\}$ and, consequently, $e \hat{\theta} e^{\prime}$. This implies that $\operatorname{dim}_{I}(G)=1$ as required.

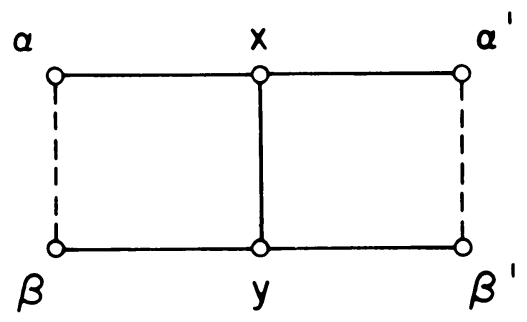

FIGURE 2 
A generalization of the tree theorem. If $T_{n}$ denotes a tree with $n$ edges, the following result was established in [GP1] (see also [GL]):

THEOREM. For the distance matrix $D\left(T_{n}\right)$ of $T_{n}$,

$$
\operatorname{det}\left(D\left(T_{n}\right)\right)=(-1)^{n} n 2^{n-1}
$$

independent of the structure of $T_{n}$.

The next result we give generalizes (2) and gives a geometrical explanation for the factor $2^{n-1}$. Consider the set $Q^{n}$ of vertices of the (usual) unit $n$-cube in $\mathbf{R}^{n}$,

$$
Q^{n}=\{0,1\}^{n}=\left\{\bar{a}=\left(a_{1}, \ldots, a_{n}\right): a_{k}=0 \text { or } 1,1 \leqslant k \leqslant n\right\} .
$$

There is a natural metric $d_{H}$ on $Q^{n}$, called the Hamming metric, given by

$$
d_{H}\left(\left(a_{1}, \ldots, a_{n}\right),\left(b_{1}, \ldots, b_{n}\right)\right)=\sum_{k=1}^{n}\left|a_{k}-b_{k}\right|,
$$

i.e., the distance between $\bar{a}$ and $\bar{b}$ in $Q^{n}$ is just equal to the number of coordinate positions in which they differ. Let us call a set $S \subseteq Q^{n}$ full dimensional if the convex hull of $S$ has positive $n$-dimensional volume.

THEOREM 4. If $\left\{\bar{a}_{0}, \ldots, \bar{a}_{n}\right\}$ is any full-dimensional set of $n+1$ points of $Q^{n}$, then

$$
\operatorname{det}\left(d_{H}\left(\bar{a}_{i}, \bar{a}_{j}\right)\right)=(-1)^{n} n \cdot 2^{n-1} \text {. }
$$

Proof. For $\bar{a}_{i}=\left(a_{i 1}, \ldots, a_{i n}\right)$ write $a_{i k}=\frac{1}{2}+\frac{1}{2} \alpha_{i k}$ where $\alpha_{i k}= \pm 1$. Thus

$$
\begin{aligned}
d_{H}\left(\bar{a}_{i}, \bar{a}_{j}\right) & =\sum_{k=1}^{n}\left|a_{i k}-a_{j k}\right|=\frac{1}{2} \sum_{k=1}^{n}\left|\alpha_{i k}-\alpha_{j k}\right| \\
& =\frac{1}{2} \sum_{k=1}^{n}\left(1-\alpha_{i k} \alpha_{j k}\right)=\frac{1}{2}\left(n-\bar{\alpha}_{i} \cdot \bar{\alpha}_{j}\right),
\end{aligned}
$$

where $\bar{\alpha}_{i} \cdot \bar{\alpha}_{j}$ denotes the inner product of the vectors $\bar{\alpha}_{i}=\left(\alpha_{i 1}, \ldots, \alpha_{i n}\right)$ and $\bar{\alpha}_{j}=$ $\left(\alpha_{j 1}, \ldots, \alpha_{j n}\right)$. It follows from elementary linear algebra that for any square matrix $M=\left(m_{i j}\right)$, if $J$ is the square matrix with all entries equal to 1 , then

$$
\operatorname{det}(M+x J)=\operatorname{det} M+x \operatorname{det}\left(m_{i j}-m_{1 j}-m_{i 1}+m_{11}\right) .
$$

Thus, from (4) we have

$$
\begin{gathered}
\operatorname{det}\left(d_{H}\left(a_{i}, a_{j}\right)\right)=\operatorname{det}\left(\frac{1}{2}\left(n-\bar{\alpha}_{i} \cdot \bar{\alpha}_{j}\right)\right)=(-2)^{-(n+1)} \operatorname{det}\left(\bar{\alpha}_{i} \cdot \bar{\alpha}_{j}-n\right) \\
=(-2)^{-(n+1)}\left\{\operatorname{det}\left(\bar{\alpha}_{i} \cdot \bar{\alpha}_{j}\right)-n \operatorname{det}\left(\bar{\alpha}_{i} \cdot \bar{\alpha}_{j}-\bar{\alpha}_{0} \cdot \bar{\alpha}_{j}-\bar{\alpha}_{i} \cdot \bar{\alpha}_{0}+\bar{\alpha}_{0} \cdot \bar{\alpha}_{0}\right)\right\} \\
=(-2)^{-(n+1)}\left\{\operatorname{det}\left(\bar{\alpha}_{i} \cdot \bar{\alpha}_{j}\right)-n \operatorname{det}\left(\left(\bar{\alpha}_{i}-\bar{\alpha}_{0}\right) \cdot\left(\bar{\alpha}_{j}-\bar{\alpha}_{0}\right)\right)\right\} .
\end{gathered}
$$

The determinants which appear in (6) of the form $\operatorname{det}\left(\bar{x}_{i} \cdot \bar{x}_{j}\right)$, called Gramians, occur frequently in linear algebra. One of their particularly useful properties is the following.

FACT (see [Ga]). For a set of vectors $\bar{x}_{1}, \ldots, \bar{x}_{n}$ in $\mathbf{R}^{m}$, the Gramian $\operatorname{det}\left(\bar{x}_{i} \cdot \bar{x}_{j}\right)$ is just the square of the ( $n$-dimensional) volume of the parallelepiped spanned by the $\bar{x}_{k}$. 
In particular, since the $n+1 \bar{\alpha}_{k}$ 's in (6) all lie in $\mathbf{R}^{n}$, then $\operatorname{det}\left(\bar{\alpha}_{j} \cdot \bar{\alpha}_{j}\right)=0$. On the other hand, the vectors $\bar{\alpha}_{k}-\bar{\alpha}_{0}$ by hypothesis span an $n$-dimensional space. It is clear that the parallelepiped they span has width 2 in each dimensional (since all $\alpha_{i k}= \pm 1$ ), and consequently, has volume $2^{n}$.

Thus, continuing (6), we obtain

$$
\operatorname{det} d_{H}\left(\bar{\alpha}_{i}, \bar{\alpha}_{j}\right)=(-2)^{-(n+1)}\left(0-n \cdot\left(2^{n}\right)^{2}\right)=(-1)^{n} n \cdot 2^{n-1}
$$

and the theorem is proved.

Symmetric differences. It is often natural to interpret binary $n$-tuples as characteristic functions on the set $[n]=\{1, \ldots, n\}$ so that each $\bar{a}=\left(a_{1}, \ldots, a_{n}\right)$ corresponds to a subset $S(\bar{a}) \subseteq[n]$ by $k \in S(\bar{a}) \Leftrightarrow a_{k}=1$. With this association it is easy to see that $d_{H}(\bar{a}, \bar{b})=|S(\bar{\alpha}) \Delta S(\bar{b})|$, where $X \Delta Y$ denotes the symmetric difference $X \backslash Y$ $\cup Y \backslash X$ of $X$ and $Y$.

Let us say that a family of subsets in $[n]$ is full dimensional if the corresponding $n$-tuples are. We can restate Theorem 4 in these terms.

TheOREM 5. Suppose $\left\{S_{0}, \ldots, S_{n}\right\}$ is a full-dimensional family of subsets of $[n]$. Then

$$
\operatorname{det}\left(\left|S_{i} \Delta S_{j}\right|\right)=(-1)^{n} n \cdot 2^{n-1} .
$$

The advantage of this formulation is that it can be readily extended to the following more general situation. Suppose $\mu$ is a discrete measure on $2^{[n]}$, i.e.,

$$
\begin{array}{ll}
\mu(k) \geqslant 0, & k \in[n], \\
\mu(X)=\sum_{x \in X} \mu(x), & X \subseteq[n] .
\end{array}
$$

THEOREM 6. Suppose $\left[S_{0}, \ldots, S_{n}\right]$ is a full-dimensional family of subsets of $[n]$. Then

$$
\operatorname{det}\left(\mu\left(S_{i} \Delta S_{j}\right)\right)=(-1)^{n} 2^{n-1} \sum_{k} \mu(k) \prod_{k} \mu(k) .
$$

The proof of (8), which we will not give here, depends upon an extension of the following result:

THEOREM [GHH]. If a connected graph $G$ has blocks, i.e., maximal 2-connected subgraphs, $G_{1}, \ldots, G_{r}$, then

(i) $\operatorname{cof}(G)=\prod_{k=1}^{r} \operatorname{cof}\left(G_{k}\right)$,

(ii) det $D(G)=\sum_{k=1}^{r} \operatorname{det}\left(D\left(G_{k}\right)\right) \prod_{i \neq k} \operatorname{cof}\left(G_{i}\right)$,

where $\operatorname{cof}(H)$ denotes the sum of the cofactors of the distance matrix $D(H)$ of the graph $H$.

Note that this result immediately implies (2) since any tree $T_{n}$ with $n$ edges has exactly $n$ blocks, each being a single edge $K_{2}$ having $\operatorname{det}\left(D\left(K_{2}\right)\right)=-1, \operatorname{cof}\left(K_{2}\right)=2$. Of course, when $\mu$ is just the counting measure, i.e., $\mu(k)=1$ for all $k \in[n]$, then (8) reduces to $(7)$.

Embedding into $n$-cubes. The cartesian product $K_{2}^{n}=\prod_{i=1}^{n} K_{2}$ (where $K_{2}$ denotes the complete graph on two points) is usually called the $n$-cube in the graph theory 
literature. The induced metric $d_{K_{2}^{n}}$ on $K_{2}^{n}$ is just the Hamming metric $d_{H}$. The problem of characterizing those $G$ for which $G \stackrel{I}{\rightarrow} K_{2}^{N}$ for some $N$ was first settled by Djokovič [Dj].

It follows from the preceding results that $G \stackrel{I}{\rightarrow} K_{2}^{n}$ for some $n$ iff

(i) $G$ is bipartite.

(ii) For all $e=\{x, y\} \in E$, if $a, b, c \in V$ such that $a x<a y, b x<b y$, and $a b=$ $a c+b c$, then $c x<c y$.

Since the distance matrix $D(G)$ for any (connected) graph $G$ is real and symmetric, then $D(G)$ has all real eigenvalues. Let $n_{+}(G)$ and $n_{-}(G)$ denote the number of positive and negative eigenvalues, respectively, of $D(G)$.

THEOREM 7. If $G \stackrel{I}{\rightarrow} K_{2}^{m}$ for some $m$, then

$$
\operatorname{dim}_{I}(G)=n_{-}(G) \text {. }
$$

Proof. Suppose $\lambda: G \stackrel{I}{\rightarrow} K_{2}^{m}$ is an isometry, say $\lambda(v)=\left(\lambda(v)_{1}, \ldots, \lambda(v)_{m}\right)$ for $v \in V(G)$. Since

$$
d_{G}\left(v, v^{\prime}\right)=d_{H}\left(\lambda(v), \lambda\left(v^{\prime}\right)\right)=\sum_{k=1}^{m} d_{H}\left(\lambda(v)_{k}, \lambda\left(v^{\prime}\right)_{k}\right),
$$

then if we define

$$
A_{k}:=\left\{v \in V: \lambda(v)_{k}=0\right\}, \quad B_{k}:=\left\{v \in V: \lambda(v)_{k}=1\right\},
$$

we have the basic decomposition

$$
\sum_{v, v^{\prime} \in V(G)} d_{G}\left(v, v^{\prime}\right) x_{v} x_{v^{\prime}}=\sum_{k=1}^{m}\left(\sum_{a \in A_{k}} x_{a}\right)\left(\sum_{b \in B_{k}} x_{b}\right) .
$$

We can rewrite $(10)$ as

$$
\sum_{v, v^{\prime} \in V(G)} d_{g}\left(v, v^{\prime}\right) x_{v} x_{v^{\prime}}=\frac{1}{4} \sum_{k=1}^{m}\left\{\left(\sum_{v \in V(G)} x_{v}\right)^{2}-\left(\sum_{a \in A_{k}} x_{a}-\sum_{b \in B_{k}} x_{b}\right)^{2}\right\}
$$

which expresses the quadratic form on the left-hand side as a sum of one positive square and $m$ negative squares. Thus, by Sylvester's "law of inertia",

$$
n_{+}(G) \leqslant 1, \quad n_{-}(G) \leqslant m .
$$

In fact, $n_{+}(G)=1$ since $D(G)$ has trace 0 .

Now, on one hand,

$$
\operatorname{rank}(D(G))=n_{-}(G)+n_{+}(G)=n_{-}(G)+1 \leqslant m+1 .
$$

On the other hand, since $G$ is connected, there must exist $v_{0}, v_{1}, \ldots, v_{m} \in V(G)$ such that the set $\left\{\lambda\left(v_{0}\right), \lambda\left(v_{1}\right), \ldots, \lambda\left(v_{m}\right)\right\}$ is full dimensional in $K_{2}^{m}$. Thus the submatrix $\left(d_{G}\left(v_{i}, v_{j}\right)\right)=\left(d_{H}\left(\lambda\left(v_{i}\right), \lambda\left(v_{j}\right)\right)\right)$ is nonsingular (by Theorem 4), and so $\operatorname{rank}(D(G))$ $\geqslant m+1$. Consequently, $\operatorname{rank}(D(G))=m+1$, and $n_{-}(G)=m=\operatorname{dim}_{I}(G)$ which proves the theorem.

We note that it follows from these considerations, for example, that if $G \stackrel{I}{\rightarrow} K_{2}^{m}$, then $\operatorname{det}(D(G)) \neq 0$ iff $G$ is a tree. 
Concluding remarks. The problem of embedding graphs isometrically into other graphs is a special case of the more general topic of embedding (finite) metric spaces isometrically into other metric (or semimetric) space. This topic has an extensive literature, some of which can be found in [ADz1, ADz2, Av3, K2, K3]. Of course, many of these more general results impinge on our studies. For example, it follows from these considerations that if $G \stackrel{I}{\rightarrow} K_{3}^{m}$ (or indeed, if $G \stackrel{I}{\rightarrow} H^{m}$ for any graph $H$ with at most four vertices), then $n_{+}(G)=1$. The reason for this is as follows.

Let us say that an $n$ by $n$ distance matrix $D=\left(d_{i j}\right)$ is of negative type if

$$
x_{1}+\cdots+x_{n}=0, \quad x_{k} \in \mathbf{R} \Rightarrow \sum_{i, j} d_{i j} x_{i} x_{j} \leqslant 0 .
$$

Similarly, call $D$ hypermetric if

$$
x_{1}+\cdots+x_{n}=1, \quad x_{k} \in \mathbf{Z} \Rightarrow \sum_{i, j} d_{i j} x_{i} x_{j} \leqslant 0 .
$$

Although (14) and (15) are similar, (15) is actually much stronger (see [K3]). Not only does it imply (14) but also that the space actually satisfies the triangle inequality (and many stronger related ones), something that (14) does not do. It is not hard to show that

$$
D \text { is of negative type } \Rightarrow n_{+}(D)=1 .
$$

On the other hand, Winkler [Win] has recently found a graph $G$ for which $n_{+}(G)=1$ but $D(G)$ is not of negative type.

It turns out that the properties of $l_{1}$-embeddability, hypermetricity and negative type are preserved under taking products, factors and isometric subsets. Thus

$K_{3}$ is of negative type (easy to check) $\Rightarrow K_{3}^{m}$ is of negative type

$$
\Rightarrow G \stackrel{I}{\rightarrow} K_{3}^{m} \text { is of negative type } \Rightarrow n_{+}(G)=1 .
$$

In fact, it can be shown that $G \stackrel{I}{\rightarrow} K_{3}^{m}$ for some $m$ iff the relation $\theta$ is transitive, i.e., $\boldsymbol{\theta}=\hat{\boldsymbol{\theta}}$.

An interesting observation (due to H. J. Landau [L]) is the following. Suppose $X$ is a semimetric space with distance matrix $D$. Let $D^{(k)}$ denote the distance matrix corresponding to the product space $X^{k}$. As just remarked, if $X$ is of negative type, then so is $X^{k}$ and, consequently, $n_{+}\left(D^{(k)}\right)=1$ for any $k$. It turns out that the converse holds. In fact, it can be shown [L] that if $n_{+}\left(D^{(2)}\right)=1$, then this already implies that $X$ must be of negative type.

It was suggested at one time by Deza [Dez2] that hypermetricity might be a sufficient condition for isometric embeddability into $l_{1}$ (i.e., $\mathbf{R}^{m}$ with $d(\bar{x}, \bar{y})=$ $\left.\sum_{i}\left|x_{i}-y_{i}\right|\right)$. This was shown not to be the case by Assouad [As1] and also by Avis [Av3], who proved that the graph $K_{7}-P_{3}$ is hypermetric but not isometrically embeddable into $l_{1}$. However, it is true [Dez1, K1] that hypermetricity is a necessary condition for $l_{1}$-embeddability. In the same spirit it is easy to show that the graph $K_{5}-K_{3}$ is of negative type but not hypermetric (see [ADz2]). 
Finally, we arrive at the graph $K_{3,2}$, which is exceptional in several respects. Since $n_{+}\left(K_{3,2}\right)=2, K_{3,2}$ is not of negative type and therefore not isometrically embeddable into any $K_{2}^{m}$ or even $\mathbf{R}^{n}$. In fact, it is not even a subgraph of $K_{2}^{m}$.

At present no necessary and sufficient conditions are known for a graph to be $l_{1}$-embeddable, hypermetric or of negative type. It would seem fruitful to study the characteristic polynomials of the associated distance matrices of various spaces rather than just the signs of the eigenvalues. This has been initiated for trees in [EGG and GL]. It seems likely that our understanding of this whole general area would increase substantially if the corresponding results were known for graphs more general than trees, e.g., for $G \stackrel{I}{\rightarrow} K_{2}^{m}$.

ACKNOWLEDGEMENTS. The authors wish to acknowledge the valuable discussions they have had on the preceding topics with H. J. Landau and H. S. Witsenhausen.

\section{REFERENCES}

[As1] P. Assouad, Un espace hypermétrique non plongeable dans un espace $L^{1}, \mathrm{C}$. R. Acad. Sci. Paris Sér. A 285 (1977), 361-363.

[As2] _ Plongements isométrique dans $L^{1}:$ Aspect analytique, Sém. d'Initiation à l'Analyse (Paris 6), exposé no. 14, 1979-1980, Univ. of Paris VI.

[As3] __ Sur les inequalités valides dans $L^{1}$, Preprint.

[ADe1] P. Assouad and C. Delorme, Graphs plongeables dans $L^{1}$, C. R. Acad. Sci. Paris 291 (1980), 369-372.

[ADe2] , Distances sur les graphes et plongements dans $L^{1}$. I, II, Preprints.

[ADz1] P. Assouad and M. Deza, Espaces métriques plongeables dans un hypercube, Ann. Disc. Math. 8 (1980), 197-210.

[ADz2] _ Metric subspaces of $L^{1}$, Preprint.

[Av1] D. Avis, Hamming metrics and facets of the Hamming cone, Tech. Report SOCS-78.4, School of Comp. Sci., McGill University, 1978.

[Av2] __ Extremal metrics induced by graphs, Ann. Dis. Math. 8 (1980), 217-220.

[Av3] __ Hypermetric spaces and the Hamming cone, Canad. J. Math. 33 (1981), 795-802.

[BM] J. A. Bondy and U. S. R. Murty, Graph theory and applications, American Elsevier, New York, 1976.

[BGK] L. H. Brandenburg, B. Gopinath and R. P. Kurshan, On the addressing problem of loop switching, Bell System Tech. J. 51 (1972), 1445-1469.

[Dew] A. K. Dewdney, The embedding dimension of a graph, Ars Combin. 9 (1980), 77-90.

[Dez1] M. Deza (M. E. Tylkin), On Hamming geometry of unitary cubes, Dokl. Akad. Nauk. SSR 134 (1960), 1037-1040. (Russian)

[Dez2] _ Realizability of matrices of distances in unitary cubes, Problemy Kibernet. 7 (1962), 31-42. (Russian)

[Dj] D. Z. Djokovič, Distance preserving subgraphs of hypercubes, J. Combin. Theory Ser. B 14 (1973), 263-267.

[EGG] M. Edelberg, M. R. Garey and R. L. Graham, On the distance matrix of a tree, Discrete Math. 14 (1976), 23-29.

[F] V. Firsov, Isometric embedding of a graph in a Boolean cube, Kibernetica (Kiev) 1 (1965), 95-96. (Russian)

[Ga] F. R. Gantmacher, The theory of matrices, vol. I, Chelsea, New York, 1959.

[GP1] R. L. Graham and H. O. Pollak, On the addressing problem for loop switching, Bell System Tech. J. 50 (1971), 2495-2519.

[GP2] __ On embedding graphs in squashed cubes, Graph Theory and Applications, Lecture Notes in Math., vol. 303, Springer-Verlag, New York, 1972, pp. 99-110.

[GHH] R. L. Graham, A. J. Hoffman and H. Hosoya, On the distance matrix of a directed graph, J. Graph Theory 1 (1977), 85-88.

[GL] R. L. Graham and L. Lovász, Distance matrix polynomials of trees, Adv. in Math. 29 (1978), 60-88. 
[K1] J. B. Kelley, Metric inequalities and symmetric differences, in Inequalities. II (O. Shisha, Ed.), Academic Press, New York, 1970, pp. 193-212.

[K2] __ Hypermetric spaces and metric transforms, in Inequalities. II (O. Shisha, Ed.), Academic Press, New York, 1970, pp. 149-159.

[K3] __ Hypermetric spaces, Lecture Notes in Math., Vol. 490, Springer-Verlag, New York, 1975, pp. 17-31.

[L] H. J. Landau, Personal Communication.

[Win] P. M. Winkler, On graphs which are metric spaces of negative type (in preparation).

[Y] A. C-C. Yao, On the loop switching addressing problem, SIAM J. Comput. 7 (1978), 515-523.

Mathematical Sciences Research Center, AT \& T, Bell laboratories, Murray Hill, New JERSEY 07974

Department of Mathematics, Emory University, Atlanta, Georgia 30322 